\title{
EL RETO TECNOLÓGICO PARA LA SOSTENIBILIDAD DE LOS MASSIVE OPEN ONLINE COURSES (MOOC)
}

\author{
Technological Challenge for Massive Open Online Courses Sustainability
}

O reto tecnológico para a sustentabilidade dos massive open online courses

RECIBIDO:21 DE JULIO DE 2015

es

\section{RESUMEN}

La tecnología es, sin lugar a dudas, uno de los elementos determinantes para la sostenibilidad y el futuro del modelo de cursos MOOC (massive open online course). Los principios en los que se sustenta el movimiento son difíciles de garantizar sin el apoyo de las herramientas y los recursos tecnológicos. Sin una apuesta decida por un modelo tecnológico fuerte no se garantizará un adecuado tratamiento pedagógico, tutorial y evaluativo de los cursos y, por supuesto, el principio de masividad seguirá siendo una utopía desde unos parámetros de calidad pedagógica aceptables. En este artículo, analizamos los modelos tecnológicos venideros y su aplicación en los cursos MOOC. En un futuro inmediato será necesario que se tomen en cuenta nuevas estrategias de aprendizaje digital por medio de los content curator, las anotaciones en línea, los programas de categorización y filtrado de la información (sistemas recomendadores), los algoritmos de aprendizaje y los sistemas tutoriales inteligentes y autoadaptativos.

PaLABRAS ClAVE: MOOC, educación, tecnología, sostenibilidad.

\section{EVALUADO:12 DE AGOSTO DE 2015}

Esteban Vázquez Cano (España) Universidad Nacional de Educación a Distancia (UNED) evazquez@edu.uned.es

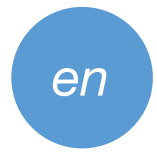

ABSTRACT

Technology is without a doubt one of the decisive elements for sustainability and the future of the MOOC model. The principles on which the movement is based are hard to guarantee without the support of technological tools and resources. It would be impossible to ensure the correct pedagogical, tutorial, and evaluative treatment of the courses if we didn't have a strong technological model. Besides, the principle of massiveness would still be a utopia from acceptable educational quality parameters. This article analyzes future technological models and their application to MOOC courses. In an immediate future, it will be necessary to keep into account new digital learning strategies through content curators, online notes, categorization programs, information filters (proposing systems), learning algorithms, and intelligent and self-adaptive tutorial systems.

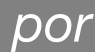

\section{RESUMO}

A tecnologia é, sem dúvidas nenhuma, um dos elementos determinantes para a sustentabilidade e o futuro do modelo de cursos MOOC (massive open online course). Os princípios nos que se sustenta o movimento são difíceis de garantir sem o apoio das ferramentas e os recursos tecnológicos. Sem uma aposta decida por um modelo tecnológico forte no se garantirá um adequado tratamento pedagógico, tutorial e avaliativo dos cursos e, por suposto, o princípio de massividade seguirá sendo uma utopia desde uns parâmetros de qualidade pedagógica aceitáveis. Em este artigo, analisamos os modelos tecnológicos por vir e a sua aplicação nos cursos MOOC. Em um futuro imediato será necessário que se tomem em conta novas estratégias de aprendizado digital por meio dos content curator, as anotações em linha, os programas de categorização e filtrado da informação (sistemas recomendadores), os algoritmos de aprendizado e os sistemas tutoriais inteligentes e auto adaptativos.
KEYWORDS: MOOC, education, technology, sustainability.
PALAVRAS CHAVE: MOOC, educação, tecnologia, sustentabilidade. 


\section{INTRODUCCIÓN}

En la concepción de Stephen Downes, un MOOC (massive open online course) es un conjunto de conexioVerónica Sánchez García I nes entre gentes, ideas: es una cultura, un idioma, una forma de ver el aprendizaje. Subrayando este aspecto, Downes (2012) define las siglas MOOC de la siguiente manera:

1. Es masivo no como resultado (como lo es un canal de TV, una emisora de radio, etc., que llegan a una gran cantidad de gente), sino que es masivo, porque tiene conexión e interactividad entre muchas personas.

2. Es abierto: lo cual implica gratuidad y que sea libre, que se pueda ir y venir.

3. Es en línea, porque se crea conexión entre diferentes personas del mundo a través de la red.

4. Es un curso, no una comunidad, ni un evento, ni un ambiente, sino que, como los cursos, comienza, transcurre y termina. Es, pues, una red temporal.

Teniendo en cuenta que el debate en torno a los MOOC no tiene siempre en cuenta estas características, Downes (2012) establece tres tipos de MOOC:

1. Los que se basan en contenidos, donde se aprende sobre un tema y hay poca interactividad.

2. Los MOOC basados en redes, como las que propone él mismo, donde no hay una ruta predefinida, puesto que cada uno escoge lo que quiera. De manera que, aunque sea masivo, es también personal, ya que cada uno tiene una experiencia diferente durante el curso.

3. Los MOOC híbridos, que se basan en la idea de tarea, incluyen una red, pero basada en un contenido.

4.

Asimismo describe qué elementos básicos debe tener un curso de estas características:

Panorama I pp. 51-50I

Volumen 91

Número 17।
4. Ambiente en línea para interaccionar entre los participantes.

Aunque estas pueden considerarse las líneas definitorias de lo que puede ser considerado un MOOC, con su

1. Wiki, donde se planifica el curso (por semanas, temas, etc.).

2. Lista de correo para suscribirse.

3. Blog del curso, para que los miembros reflexionen día a día sobre el propio aprendizaje.

puesta en marcha han surgido muchos cuestionamientos sobre su funcionamiento pedagógico y sobre las posibilidades que la tecnología ofrece para garantizar que la formación masiva en abierto pueda ser de calidad (Daniel, Vázquez-Cano y Gisbert, 2015; Vázquez-Cano y López Meneses, 2014; Vázquez-Cano, 2013; Vázquez-Cano, López Meneses y Sarasola, 2013). Lo que no cabe duda es de que los MOOC plantean una realidad y son la respuesta a algunas de las características más destacables de la sociedad emergente: la posibilidad de acceso abierto y sin mediación a recursos del conocimiento por medio de la tecnología, el bucle demanda/ devaluación de títulos, la carestía creciente de los estudios y la burbuja universitaria, entre otros (Zapata-Ros, 2013, p. 23).

\section{LA TUTORÍA AUTOMATIZADA PARA EL SEGUIMIENTO DE LA MASIVIDAD}

La interacción (Zapata-Ros, 2012) considerada cómo la inmediatez y el ajuste de la respuesta y de las contrarrespuestas a las preguntas, las intervenciones o las demandas que se producen, y su carácter de mediación, en este caso entre profesores y alumnos, ha desaparecido. Para cumplir su gran promesa de ofrecer una mejor educación que optimice la calidad del aprendizaje por parte del estudiante, los MOOC tendrán que explotar los últimos avances tanto en procesamiento de datos a gran escala como en aprendizaje de máquina. Además, para ofrecer clases complejas y de manera simultánea a miles de personas, tendrán que recurrir a altos niveles de automatización; por caso, muchas de las tareas más demandantes en tiempo, tradicionalmente realizadas por profesores y monitores, como corregir exámenes, moderar discusiones, etc., deberán ser ejecutadas por computadores.

La optimización del tiempo docente mediante herramientas y recursos en red que fomenten la escabilidad es una necesidad para un adecuado tratamiento de la masividad. En este epígrafe, presentaremos algunas herramientas que pueden resultar útiles para el desarrollo tutorial de cursos MOOC. Entre ellas, queremos destacar las siguientes (Vázquez-Cano, López Meneses y Barroso, 2015):

\section{CALIBRATEd PEER REVIEW}

Es un sistema que se experimentó en la Universidad de California y que en la actualidad se ha extendido en 
muchas instituciones de educación superior. Se aplica principalmente en el desarrollo de reflexiones por escrito que precisan retroalimentación por otros compañeros de forma que se mejoren la propia redacción y los argumentos expuestos previamente (Sadler y Good, 2006). El proceso se ha redefinido desde entonces y en la actualidad incorpora cuatro fases: en la primera, los estudiantes redactan y envían un ensayo o tema en el formato que le pide el tutor-profesor. En una segunda fase, los estudiantes evalúan de forma aleatoria tres trabajos de forma contrastada con el uso de una rúbrica creada al efecto. En la tercera fase, y una vez validada la rúbrica, cada estudiante recibe tres trabajos anónimos de otros estudiantes y se evalúan con la rúbrica. En la cuarta y última fase, cada estudiante recibe las evaluaciones de sus compañeros y los comentarios realizados.

\section{FACULTY-DIRECTED "STUDENT-SOURCING" OF RESPON-} SES TO STUDENT QUESTIONS

Algunos estudiantes esperan que los tutores estén conectados para cuando necesitan una aclaración o ayuda. Los avances en el filtrado y la selección de correos electrónicos crowdsourcing pueden ser una herramienta muy útil para facilitar así la respuesta por los propios alumnos y se hace pública en un lugar de la plataforma al efecto (preferiblemente una wiki, ya que permite la edición de cada estudiante y la creación colectiva de la respuesta). En esta wiki los profesores-tutores pueden formular preguntas tipo que desarrollen el programa y sean co-respondidas por los propios estudiantes, quienes retroalimentan y cuestionan determinados matices que luego terminarán con una intervención final del profesor, donde se postulen los aciertos y errores de los estudiantes. Una variante de este sistema se puede construir con preguntas y respuestas anónimas de los estudiantes. El sistema mejora sustancialmente cuando se puede participar desde diferentes soportes multiplataforma como aplicaciones para móviles y tabletas.

\section{JUST-IN-TIME TEACHING}

En la actualidad, se conoce con el sintagma inglés Flipped Classroom donde la introducción o pretexto para el desarrollo se produce con la introducción de otro tópico paralelo sobre el que se pide al estudiante que reelabore una propuesta o crítica. Khan Academy ha explotado este sistema con una colección de más de 4000 videos de unos 10 minutos de duración, que dependiendo de cada curso son utilizados para favorecer este tipo de clase invertida (EdSurge, 2012). No se trata de añadir videos para alargar la clase, sino de organizar la clase para que sea más autoguiada por el estudiante. Para ello, el profesor-tutor debe optar por el sistema Just-in-Time Teaching, que consiste en proporcionar al estudiante la adquisición de destrezas que le sirvan a lo largo de sus estudios y en el mundo laboral. Para ello, el estudiante piensa y reelabora preguntas propuestas por el profesor antes de iniciar el curso, que son autoevaluadas y le proporcionan a aquel una retroalimentación efectiva sobre qué aspectos requieren mayor atención y reflexión. Este sistema combina datos e interacciones de los estudiantes, y ha sido utilizado con éxito en muchas asignaturas universitarias (Novak, 1999 y Middendorf, 2004) y para el desarrollo de competencias y destrezas que precisan un trabajo continuado en el tiempo. Uno de los aspectos más determinantes para su desarrollo eficiente es el planteamiento de preguntas adecuadas desde el inicio del curso, que integren la reflexión, el análisis y la interacción entre estudiantes (Watkins y Mazur, 2010).

\section{LOS SISTEMAS RECOMENDADORES}

Los sistemas recomendadores son uno de los campos emergentes de la inteligencia artificial, su desarrollo en el ámbito educativo promete potenciar y sostener el desarrollo de tendencias innovadoras en la educación, lo cual permitirá una "masificación personalizada”, en la que cada estudiante es atendido considerando sus particularidades y estilos de aprendizaje. De forma general, se puede definir un sistema recomendador como un programa que propone o sugiere temas, actividades $o$ productos según las preferencias de los usuarios (VelezLangs y Santos, 2006). Pueden abarcar dos áreas, la primera es predecir si un producto va a ser del agrado de un usuario, y la segunda es recomendar productos según los gustos y las preferencias del usuario (Sarwar et al., 2001). La aplicación de estos conceptos en el ámbito educativo y concretamente de los MOOC implica que el ambiente de aprendizaje (plataforma) sea capaz de recomendar recursos y actividades según las preferencias y los estilos de aprendizaje de los estudiantes, asimismo implica considerar de forma particular las necesidades de cada persona haciendo efectivo el concepto de personalización, y permite atender a grandes cantidades de estudiantes, puesto que las recomendaciones son hechas por un componente artificial y no por el docente. Los sistemas recomendadores se definen también como
El reto tecnológico para la

sostenibilidad de los massive open online coursez (MOOC)
I Panorama

I pp. 51-60

I Volumen 9

I Número 17

| Julio-diciembre | 2015 
elementos inteligentes de filtrado de información que proporcionan recomendaciones a la medida sobre productos destinados a un usuario (Peña y Riffo, 2008), cuentan con mecanismos para sugerir servicios, objetos Verónica o personas que son de interés en un contexto especíSánchez fico (Alejandres, González y Vargas, 2011), lo cual García I implica que estos sistemas requieren filtrar o seleccionar información de los usuarios para, según ella, realizar recomendaciones. La interacción entre el usuario y el entorno virtual de aprendizaje incluye un conjunto de relaciones, en las que intervienen los componentes típicos de un sistema, por lo que se trata de un proceso dinámico e iterativo en el que de forma constante se obtiene información del estudiante, la cual se procesa y posteriormente se presentan recomendaciones.

El procesamiento que se da a la información registrada incluye, entre otros, algoritmos de filtrado colaborativo (Huang, Lu y Duan, 2011), los que permiten recomendar ítems a usuarios con perfiles similares que poseen los mismos gustos y preferencias, así en este enfoque es posible identificar elementos basados en las opiniones de los "usuarios" que son "similares" para poder brindar la recomendación; algoritmos basados en contenidos (Pazzani y Billsus, 2007), que se emplean por sistemas diseñados para recomendar ítems que consideran las características individuales de los usuarios; algoritmos híbridos (Chen et al., 2012), que combinan los dos métodos de recomendación, es decir, incluyen características de los sistemas basados en contenidos dentro del enfoque colaborativo.

Los avances en el campo de la personalización del aprendizaje son significativos, por lo que distintos proyectos han resultado exitosos, así tenemos el proyecto europeo EU4ALL dirigido a estudiantes con discapacidad, este proyecto contempla el desarrollo de un sistema recomendador como una forma de brindar apoyo adaptativo al proceso de aprendizaje. En Drachsler, Hummel

Panorama I pp. 51-50।

Volumen 9 ।

Número 17 I Julio-diciembre I 2015। y Koper (2008), se describen los requerimientos, las técnicas y el modelo inicial para un sistema recomendador personal para estudiantes en redes de aprendizaje para toda la vida, asimismo proponen una combinación de técnicas de recomendación basadas en reglas pedagógicas para realizar recomendaciones personalizadas de las actividades de aprendizaje en el contexto de e-learning. Por otra parte, en Vialardi et al. (2009) se presenta el uso de un sistema en el que las recomendaciones están basadas en la identificación de tipos de usuarios con la ayuda de técnicas de aprendizaje automático, para proporcionar recomendaciones apropiadas a las características individuales de los estudiantes. Asimismo Santos y Boticario (2008) presentan la experiencia de usuarios con un sistema recomendador, donde se consideran los elementos que se requieren para la definición de recomendaciones, entre ellas la identificación de las situaciones en la que es necesario ofrecerlas, las mismas que pueden determinarse según la información que se obtenga del estudiante. Otro modelo propuesto se describe en Khribi, Jemni y Nasraoui (2009), quienes describen un sistema de recomendación personalizado de objetos de aprendizaje para los estudiantes que utilizan un sistema de e-learning. Además de recomendar recursos y caminos de aprendizaje, estas técnicas se pueden emplear para mejorar la eficiencia de los sistemas educativos a todo nivel. Uno de los mayores retos de los MOOC es mejorar los índices de eficiencia terminal, motivando que sus participantes no abandonen los cursos en los cuales están inscritos. Esto abre las puertas a ramas emergentes, como la analítica del aprendizaje, minería de datos, análisis de redes sociales, entre otras.

La adecuada gestión de datos proporciona pistas que permiten anticiparse, actuando previamente para mejorar la eficiencia de un sistema educativo en su conjunto. La analítica del aprendizaje se define como el uso de modelos predictivos y otras técnicas analíticas para apoyar los objetivos institucionales y curriculares (Bach, 2010), para lo cual se requiere como insumo la información acerca del trabajo del estudiante. Entre las principales aplicaciones está la prevención de la deserción (Campbell, DeBlois y Oblinger, 2007), para lo cual se emplean alarmas que informan sobre el desempeño en un entorno virtual, las cuales permiten mejorar el rendimiento de los estudiantes en situación de riesgo (Arnold, 2010; Johnson et al., 2011). La utilización de la información para actuar en tiempo real cambiando un escenario desfavorable para el estudiante tiene un gran potencial, pues sus implicaciones permiten, en teoría, identificar deficiencias institucionales a todo nivel, desde didácticas hasta organizacionales, que una vez conocidas permiten la toma de decisiones de mejora.

En el contexto de los MOOC, aún falta mucho por trabajar el concepto de inteligencia artificial, por ello, la introducción de tal concepto en estos cursos se convierte en una oportunidad para fortalecer el proceso de enseñanza-aprendizaje que se brinda a través de estos 
cursos. Actualmente, este tipo de tecnologías están permitiendo diseñar instrumentos de aprendizaje efectivos que permiten lograr grandes beneficios. Dada la naturaleza de los MOOC, con este tipo de enfoques se podría fomentar un aprendizaje activo y personalizado centrado en el estudiante y que sea compatible con el principio de masividad.

Todos los aspectos señalados en estos cursos necesitan ser atendidos para lograr un aprendizaje más integral y eficaz. En este sentido, aplicaciones como los sistemas recomendadores (Okoye et al., 2012), que hacen las veces de tutores virtuales y que den soporte al aprendizaje del estudiante y un soporte dinámico en situaciones no cubiertas por el diseño instruccional del curso MOOC, son el camino que se debe seguir para potenciar la solución a los problemas que trae consigo el aprendizaje masivo.

Los sistemas recomendadores pueden brindar apoyo al usuario a través de sugerencias de qué acciones realizar sobre elementos disponibles en el curso, aspecto que suele ser orientado por un tutor, como sugerir al participante la realización de actividades aprendizaje, el seguimiento de rutas de navegación, la consulta de material educativo, contestar un mensaje en el foro, qué hacer ante determinado problema o tarea, o la sugerencia de cualquier otro recurso que permita potenciar y mejorar su proceso de aprendizaje (Drachsler, Hummel y Koper, 2008; Santos y Boticario, 2012). Según esto, los participantes pueden recibir ayuda en el desempeño de las tareas del curso, con el propósito de 1) mejorar el desempeño de los procesos de aprendizaje facilitando los contenidos del curso más apropiados y las rutas de aprendizaje adaptadas a las necesidades de los estudiantes $\mathrm{y}$ b) promover la colaboración entre pares (Santos y Boticario, 2012). Además, en un curso MOOC, el sistema de recomendación puede sugerir al usuario nuevos ítems o acciones, considerando la información de sus preferencias personales o las de otros usuarios con características similares.

\section{LEARNING ANALYTICS: LA MEDICIÓN DE LA ACTIVIDAD DE ESTUDIO PARA CONTROLAR EL ESFUERZO}

Los sistemas adaptativos según las herramientas digitales permiten un seguimiento del estudiante y las estadísticas que se generan facilitan al profesorado datos interesantes para modificar la didáctica, corregir lagunas y potenciar nuevas formas de aprendizaje (Johnson et al., 2011, p. 28). Se basa en un proceso de verificación de tareas realizadas por los estudiantes de forma automática, lo cual proporciona información directa al profesor-tutor de las actividades no realizadas (Lockyer, Heathcote y Dawson, 2013). La Universidad de Purdue puso en práctica esta metodología con un curso denominado Course Signals (Purdue, 2011). Este curso se diseñó para evaluar los patrones de comportamiento y su relación con el rendimiento académico para valorar si están en peligro de no alcanzar la suficiencia mínima en el desarrollo de un curso. El curso tenía dos objetivos prioritarios:

1. Proporcionar al estudiante claves e indicadores de su rendimiento en un curso, de forma que puedan adaptar su ritmo de estudio y aprendizaje (localizar recursos extras, invertir más tiempo o pedir ayuda).

2. Ayudar al profesorado a identificar el ritmo de desarrollo del curso y proporcionar pautas y sugerencias de mejora y profundización a los estudiantes.

Estos sistemas de seguimiento académico del estudiante se basan en un algoritmo que predice el éxito del estudiante a través del filtrado de una serie de indicadores previamente establecidos por el profesor del curso. Por ejemplo, el sistema calcula diferentes parámetros: lectura del material en línea, participación en foros de discusión, resultados de las autoevaluaciones, entre otros parámetros, y calcula un indicador de éxito de los estudiantes. Generalmente, se acompaña de una representación visual por colores, como los de un semáforo, que ayuda rápidamente a profesores y estudiantes a identificar el nivel de desarrollo y competencia alcanzado hasta una fecha. Asimismo, el sistema remite a profesores y alumnos un informe vía correo electrónico y mensaje de texto a un smartphone con un resumen estadístico del estado del progreso del estudiante.

En la actualidad, el área de investigación y desarrollo Learning Analytics es un campo emergente de estudio que requiere laboratorios y equipos de investigación que valoren la incidencia de los modelos y algoritmos que mejor respondan a la medición y variables que influyen en cada tipo de curso. De hecho, varias instituciones están investigando en este campo, por ejemplo la Universidad de Michigan con el proyecto Provost's Task Force on Analytics y la Universidad de Standford con Analytics Lab (MacKay, 2013).
| El reto tecnológico para la

sostenibilidad de los massive open online coursez (MOOC)
I Panorama

I pp. 51-60

I Volumen 9

I Número 17 I Julio-diciembre | 2015 


\section{AGREGACIÓN, FILTRADO Y CURACIÓN DE CONTENIDOS}

La selección, organización, gestión, presentación y distribución de recursos y noticias en la web es una necesidad en la actual sociedad de la información y la comunicación caracterizada por la abundancia temática y el intercambio de experiencias. Con los procesos de agregación, filtrado y curación de contenidos en los cursos MOOC, conseguimos ampliar la experiencia del estudiante y ser capaces de sistematizar y dar sentido a todo el flujo de información y recursos que se produce en los foros. En un interesante artículo, de Benito et al. (2013) establecen las características generales de estos tres procesos:

1. El filtrado hace una criba de información según su relación con un tema y contexto determinados. Los sistemas de filtrado son herramientas que ayudan a las personas a encontrar la información más valiosa, de forma que el poco tiempo que se emplee en leer, escuchar o ver sean los documentos más interesantes y relevantes.

2. La agregación la entendemos como la recopilación y presentación de información sobre un mismo tema y contextos determinados que previamente se habían filtrado.

3. La curación de contenidos (content curation) la entendemos como un concepto más amplio que comprendería también los procesos de filtrado y agregación, comentados anteriormente. Según Digital Curation Centre (2010), la curación digital implica mantener, conservar y añadir valor a la información encontrada durante su ciclo de vida. De acuerdo con Bhargava (2009), el curador se trata de la "persona que continuamente encuentra, agrupa, organiza y comparte el mejor y más relevante contenido sobre un tema específico en línea".

En la actualidad, el content curator es la figura que más se demanda para facilitar que los foros y la masividad adquieran valor en los cursos MOOC. Para ello, el proceso de curación debe responder de forma estructurada a una serie de fases, entre las que podemos citar:

Panorama I pp. 51-50I

Volumen 91

Número 17|

3. Valoración y selección: se evalúa la información recogida y se selecciona la que se quiere preservar a largo plazo.

4. Conversión: se transfieren los datos a un lugar, como un repositorio o archivo.
5. Preservación: se asegura la conservación de la información, tal como se obtuvo limpiando, validando y asignando metadatos de preservación.

6. Almacenamiento: se trata de guardar la información siguiendo los estándares establecidos a esos efectos.

7. Acceso, uso y reutilización: se asegura la accesibilidad posterior de la información.

8. Transformación: creación de información nueva a partir de la original. Esta transformación se puede hacer de diferentes formas, planteadas en los modelos propuestos por Bhargava (2011):

A. Agregación: curación de la información más relevante sobre un tema en particular en un único lugar.

B. Destilación: curación de la información en un formato más sencillo donde solo se compartan las ideas más importantes.

C. Elevación: curación para la identificación de una tendencia mayor a partir de breves reflexiones diarias publicadas en línea.

D. Mashup o remezcla: mezcla de contenido que ya existe para crear un nuevo punto de vista.

E. Cronología: combinación de información histórica organizada basada en la cronología para facilitar la comprensión de la evolución de un tema concreto.

\section{LAS ANOTACIONES EN LÍNEA}

El modelo audiovisual en el que se inspiran los cursos MOOC mediante visionado de videos modulares explicativos precisa de herramientas y recursos que enriquezcan el elemento audiovisual y posibiliten una mayor explotación didáctica. En este proceso, aparecen las anotaciones multimedia como un recurso productivo. Las anotaciones de video permiten añadir capas de texto, enlaces y zonas activas en un video. Las herramientas de anotación de video han emergido para ayudar a los educadores de forma más efectiva a reflexionar y analizar los procesos de enseñanza (Rich y Hannafin, 2009).

Estas herramientas permiten al usuario marcar fragmentos del video añadiendo texto y comentarios visuales, para enriquecer las explicaciones. En la actualidad, el número de herramientas de video con anotación disponible está creciendo, variando desde sofisticados programas hasta herramientas de internet. El objetivo es que los estudiantes y los educadores puedan, entre otras funciones: 
1. Etiquetar fácilmente o tomar notas en un video, hacer guías de estudio o de revisión, que son privadas o abiertas al público.

2. Compartir comentarios o participar en foros de discusión acerca de un segmento de un video.

Esta metodología ya se ha puesto en práctica en el Proyecto VITAL de la Columbia University. Vital es una plataforma de video en línea para realizar ensayos "multimedia" utilizando video, que permite citar con segmentos de video y anotaciones. El objetivo de esta herramienta es que el alumno aprenda a observar detenidamente, interpretar lo que se visualiza y desarrolle conclusiones citando el contenido del video como referencia.

\section{MODELOS DE SISTEMAS DE TUTORES INTELIGENTES $Y$ ADAPTATIVOS}

Las últimas tendencias en sistemas de seguimiento del alumnado en entornos virtuales masivos se están reorientando hacia la investigación en sistemas educativos basados en webs inteligentes y adaptativas, que van más allá al construir un modelo de los objetivos, preferencias y conocimiento de cada alumno individual y focalizan la interacción del estudiante durante su proceso de aprendizaje, con la intención de adaptarlo a sus necesidades. Estos sistemas tutores inteligentes (STI) se combinan con los sistemas hipermedia adaptativos (Vázquez-Cano y Sevillano, 2015). Un STI es capaz de guiar al alumno a lo largo de un dominio en particular del conocimiento, resolviendo durante el proceso tareas, tales como la elaboración de una estrategia de tutorización, la generación de ejercicios a la medida de las necesidades del alumno, la resolución pedagógica de estos ejercicios, así como la explicación de la solución.

Estas tareas se organizan en distintos módulos, siendo los componentes clave del STI tradicional un modelo del alumno, un modelo pedagógico, un modelo didáctico y una interfaz con la que interactúa el usuario. Dependiendo de la arquitectura del sistema, estos módulos se pueden encontrar organizados en diferentes formas. Pueden estar distribuidos y subdivididos en partes más pequeñas, funcionando como entidades, semi- o completamente autónomas, que se comunican entre sí y actúan racionalmente de acuerdo con sus percepciones del exterior y el estado de su conocimiento. Podríamos decir que esta es una arquitectura basada en agentes inteligentes. Además, existen otros tipos de tutores inteligentes, los cuales se basan en estos módulos, pero crean otros personalizados.

El alumno presenta un estilo de aprendizaje y unos conocimientos iniciales que se modifican dinámicamente a partir de la interacción con el curso. Esta información del alumno se extrae y se almacena en una base de datos para su posterior análisis mediante técnicas de minería de datos. La minería de datos realimenta al controlador del ITS, y adapta los modelos que conforman el curso MOOC.

Un sistema STI es capaz de adaptarse al ritmo de aprendizaje, ofreciendo las áreas en las que el alumno se destaca y la ratio de contenidos a medida que se adquiere mayor conocimiento. Los agentes se modelan para realizar tareas pedagógicas y distribuir eficientemente las lecciones a los alumnos. Se introduce un entorno de trabajo que captura la creación dinámica de conocimiento en el dominio de interés y el contenido de conocimiento aprendido por el alumno a lo largo del tiempo (tiempo empleado por lección, tiempo por sesión, número de revisiones, frecuencia de peticiones de ayuda, tiempo medio por página). El sistema está formado por un agente tutor que guía el proceso de aprendizaje decidiendo las acciones pedagógicas que se deben realizar, el modelo del aprendiz que contempla el estilo de aprendizaje, la comprensión de los temas, las limitaciones y el nivel de conocimientos, el agente colaborativo que busca a otros aprendices que estén tratando el mismo tema para establecer comunicación sincrónica o asincrónica y ofrecer colaboración al aprendiz y, por último, la interfaz que es el puente entre los agentes humanos y los de software. Se fundamenta el paradigma instruccional en tres modelos pedagógicos: conductismo, cognitivismo e histórico-social (cognición distribuida y aprendizaje basado en problemas).

Estos modelos se complementan con el empleo de redes neuronales que evalúan automáticamente el nivel de conocimiento metacognitivo del estudiante observando su comportamiento de navegación en línea. El modelo se implementa mediante una red neuronal multicapa realimentada (MLFF 'multi-lane free flow') formada por tres capas, que clasifica a los estudiantes a partir de datos de navegación (páginas visitadas, glosarios y transcripciones consultadas y tiempo empleado). En su aplicación a los cursos MOOC, se debería aplicar según un sistema multiagente que busca la adaptabilidad
El reto tecnológico para la sostenibilidad de los massive open online coursez (MOOC)
I Panorama

I pp. 51-60

I Volumen 9

I Número 17

I Julio-diciembre | 2015 
mediante el uso de redes neuronales. La estructura propuesta consta de un sistema gestor del curso y de un sistema inteligente multiagente que implementa todos los módulos del STI.

Verónica

Sánchez

García I

El modelo del estudiante usa lógica borrosa para determinar el grado de conocimiento adquirido por el estudiante en un tiempo dado. Para determinar la evaluación del alumno, se emplean reglas borrosas entre la evaluación del conocimiento (calidad de las respuestas y tiempo empleado) y la evaluación del comportamiento (tiempo por página, movimiento de la barra de desplazamiento, participación en chats y foros). Se emplean redes neuronales, concretamente Cuantificación de Vectores de Aprendizaje (CVA) para implementar la clasificación de patrones (los estudiantes), de modo que los agentes pedagógicos puedan adaptar correctamente el currículo.

También se aplican algoritmos genéticos mediante la versión de algoritmos evolutivos multiobjetivo para el descubrimiento de relaciones importantes a partir de datos de utilización (tiempos de acceso, aciertos y fallos, y niveles de conocimiento) de los estudiantes.

\section{CONCLUSIONES}

Uno de los mayores retos de los MOOC se centra en las posibilidades que ofrece la tecnología para atender la masividad. Es una utopía pensar que un curso que pueden realizar más de cien mil personas pueda ser atendido y desarrollado por dos o tres tutores o mediante los recursos tradicionales de la enseñanza en línea. El tratamiento integral del modelo pedagógico, de tutoría y evaluación de los cursos MOOC precisa de herramientas tecnológicas que posibiliten un tratamiento adecuado de la masividad en estos entornos formativos. En este artículo hemos analizado cómo las anotaciones en línea permiten al usuario marcar fragmentos del video añadiendo texto y comentarios visuales, para enriquecer las explicaciones de los videos que se ofrecen en los cursos MOOC. Asimismo, los programas de categorización y filtrado de la información (sistemas recomendadores) pueden brindar apoyo al usuario a través de sugerencias de qué acciones realizar sobre elementos disponibles en el curso, aspecto que suele ser orientado por un tutor, como sugerir al participante la realización de actividades de aprendizaje, el seguimiento de rutas de un mensaje en el foro, qué hacer ante determinado problema o tarea o la sugerencia de cualquier otro recurso que permita potenciar y mejorar su proceso de aprendizaje. Junto con estos sistemas recomendarores, los algoritmos de aprendizaje y los sistemas tutoriales inteligentes y autoadaptativos pueden resultar herramientas muy valiosas para la atención de un modelo eficiente en entornos formativos masivos. Un "sistema tutorial inteligente" es capaz de guiar al alumno a lo largo de un dominio en particular del conocimiento, resolviendo durante el proceso tareas, tales como la elaboración de una estrategia de tutorización, la generación de ejercicios a la medida de las necesidades del alumno, la resolución pedagógica de estos ejercicios, así como la explicación de la solución.

Por tanto, creemos que para la sostenibilidad y el éxito futuro de la formación masiva y en abierto un curso MOOC debería crearse fusionando diferentes tipos de recursos multimedia y de autogestión tecnológica y no solo tomar el video como el recurso didáctico predominante. De esta manera, se enriquece el contexto de la enseñanza-aprendizaje y se crean tipos de recursos multimedia mucho más complejos y específicos para las competencias pretendidas. Compartimos con esta última reflexión la de otros autores de que si lo que queremos es cambiar los sistemas de educación y de capacitación desde los aún vigentes, que fueron diseñados para seleccionar a los alumnos, hemos de afrontar este reto en la situación actual, porque ese es un riesgo que aún se corre con la actual configuración de los MOOC. Se ha de posibilitar un cambio a otros sistemas diseñados para maximizar el aprendizaje mediante el análisis y la adaptación de las estrategias de enseñanza a los individuos (Zapata-Ros, 2013, p. 35).

\section{REFERENCIAS BIBLIOGRÁFICAS}

1. Alejandres, H. O., González, J. G. y Vargas, B. A. (2011). Sistemas de recomendación en ambientes organizacionales: estado del arte y tendencias futuras. En IX Congreso Nacional sobre Innovación y Desarrollo Tecnológico (pp. 428-434). México.

2. Arnold, K. (2010). Signals: Applying Academic Analytics. EDUCAUSE Quarterly, 33(1). Recuperado de http://www. educause.edu/EDUCAUSE+Quarterly/ EDUCAUSEQuarterlyMagazineVolum/ SignalsApplyingAc 
3. Bach, C. (2010). Learning Analytics: Targeting Instruction, Curricula and Student Support. International Instruction on Informatics and Systemics Conference. Orlando, USA. Recuperado de http://www.iiis.org/CDs2010/CD2010SCI/ EISTA_2010/PapersPdf/EA655ES.pdf

4. Bhargava, R. (2009). Manifesto for the Content Curator: The Next Big Social Media Job of the Future? Influential Marketing Blog. Recuperado de http://www.rohitbhargava.com/2009/09/ manifesto-for-the-content-curator-the-nextbig-social-media-job-of-the-future.html

5. Campbell, J., DeBlois, P. y Oblinger, D. (2007). Academic Analytics a New Tool for a New Era. EDUCAUSE review, 42(4), 42-57.

6. Chen, W., Niu, Z., Zhao, X. y Li, Y. (2012). A Hybrid Recommendation Algorithm Adapted in E-Learning Environments. World Wide Web, $1-14$.

7. Daniel, J., Vázquez-Cano, E. y Gisbert, M. (2015). The Future of MOOCs: Adaptative Learning or Business Model? RUSC: Universities and Knowlwdge Society Journal, 12(1), 64-73.

8. de Benito Crosetti, B., Mesquida, A. D., Carrió, A. L., Juarros, V. M., García, J. M., \& Ibáñez, J. S. (2013). Agregación, filtrado y curación para la actualización docente. Pixel-Bit: Revista de medios y educación, (42), 157-169.

9. Digital Curation Centre (2010). What Is Digital Curation? Recuperado de http://www.dcc.ac.uk/ digital-curation/what-digital-curation

10. Downes, S. (2012). The Rise of MOOC. Recuperado de http://www.downes.ca/ post $/ 57911$

11. Drachsler, H., Hummel, H. G. K. y Koper, R. (2008). Personal Recommender Systems for Learners in Lifelong Learning: Requirements, Techniques and Model. International Journal of Learning Technology, 3(4), 404-423.

12. EdSurge (2012). MOOCs. Recuperado de https://www.edsurge.com/moocs

13. Huang, Z., Lu, X. y Duan, H. (2011). ContextAware Recommendation Using Rough Set Model and Collaborative Filtering. Artificial Intelligence Review, 35(1), 85-99.

14. Johnson, L., Smith, R., Willis, H., Levine, A. y Haywood, K. (2011). The 2011 Horizon Report. Austin, Texas: The New Media Consortium.

15. Khribi, M. K., Jemni, M. y Nasraoui, O. (2009). Automatic Recommendations for E-Learning Personalization Based on Web Usage Mining Techniques and Information Retrieval. Educational Technology E' Society, 12(4), 30-42.
16. Lockyer, L., Heathcote, E. y Dawson, S. (2013). Informing Pedagogical Action: Aligning Learning Analytics with Learning Design. American Behavioral Scientist, 57(10), 1439-1459.

17. Mackay, R. F. (2013). Learning Analytics at Stanford Takes Huge Leap Forward with MOOCs. Stanford Report. Recuperado de http://news.stanford.edu/news/2013/april/online-learning-analytics-041113.html

18. Marrs, K. A. y Novak, G. (2004). Just-in-Time Teaching in Biology: Creating an Active Learner Classroom Using the Internet. Cell Biology Education, 3(1), 49-61. Recuperado de http:// www.lifescied.org/content/3/1/49.short

19. Middendorf, J. (2004). Facilitating a faculty learning community using the decoding the disciplines model. New Directions for Teaching and Learning, 2004 (98), 95-107.

20. Novak, G, Patterson, E.T., Gavrin, A.D., and Christian, W. (1999). Just-In-Time Teaching: Blending Active Learning with Web Technology,Upper Saddle River, NJ: Prentice Hall. Okoye, I., Maull, K., Foster, J.y Sumner, T. (2012). Educational Recommendation in an Informal Intentional Learning System. En O. C. Santos y J. G. Boticario (eds.), Educational Recommender Systems and Technologies: Practices and Challenges (pp. 1-23).

21. Pazzani, M. y Billsus, D. (1997). Learning and Revising User Profiles: The Identification of Interesting Web Sites. Machine Learning, 27(3), 313-331.

22. Peña, F. y Riffo, R. (2008). Revisión, selección e implementación de un algoritmo de recomendación de material bibliográfico utilizando tecnología $\{j 2 E E\}$. Concepción, Chile: Universidad del Bío-Bío. Recuperado de http://cybertesis. ubiobio.cl/tesis/2008/riffo_r/doc/riffo_r.pdf

23. Purdue (2011). What Is a MOOC? Recuperado de https://www.purdue.edu/learning/ $\mathrm{blog} / \mathrm{p}=5744$

24. Rich, P. y Hannafin, M. (2009). Video Annotation Tools: Technologies to Scaffold, Structure, and Transform Teacher Reflection. Journal of Teacher Education, 60(1), 52-67.

25. Sadler, P. y Good, E. (2006). The Impact of Self-and Peer-Grading on Student Learning. Educational Assessment, 11(1), 1-31.

26. Santos, O. C. y Boticario, J. G. (2012). Affective Issues in Semantic Educational Recommender Systems. En 2nd Workshop on Recommender Systemsfor Technology Enhanced Learning (RecSysTEL 2012) (pp. 71-72). Saarbrücken, Alemania. 
27. Sarwar, B., Karypis, G., Konstan, J.y Ried1, J. (2001). Item-Based Collaborative Filtering Recommendation Algorithm. Proceedings of the Tenth International World Wide Web Conference (pp. 285-295). ACM.

Verónica

Sánchez

García I

28. Vázquez-Cano, E. (2013). The Video Article: New Reporting Format in Scientific Journals and its Integration in MOOCs. Comunicar, 41, 83-91.

29. Vázquez-Cano, E., López Meneses, E. y Sarasola, J. L. (2013). La expansión del conocimiento en abierto: los MOOCs. Barcelona: Octaedro.

30. Vázquez-Cano, E. y López Meneses, E. (2014). Los MOOC en la educación superior: la expansión del conocimiento. Profesorado: Revista de Currículum y Formación del Profesorado, 18(1), $1-12$.

31. Vázquez-Cano, E., López Meneses, E. y Barroso Osuna, J. (2015). El futuro de los MOOC: retos de la formación on-line, masiva y abierta. Madrid: Síntesis.

32. Vázquez-Cano, E. y Sevillano, M. ${ }^{\mathrm{a}}$ L. (2015). Dispositivos digitales móviles en educación: el aprendizaje ubicuo. Madrid: Narcea.

33. Velez-Langs, O. y Santos, C. (2006). Sistemas recomendadores: un enfoque desde los algoritmos genéticos. Gestión y Producción, 1(9), 23-31.

34. Vialardi, S. C., Bravo, A. J., Shafti, L. y Ortigosa, A. (2009). Recommendation in Higher Education Using Data Mining Techniques. En 2nd International Conference of Educational Data Mining (pp. 190-199).

35. Watkins, J. y Mazur, E. (2010). Just-in-Time Teaching and Peer Instruction. En Just in Time Teaching: Across the Disciplines, and Across the Academy (pp. 39-62). Stylus Publishing.

36. Zapata-Ros, M. (2012). Calidad y entornos ubicuos de aprendizaje. RED: Revista de Educación a Distancia, 31, 1-12. Recuperado de http://www.um.es/ead/red/31/zapata_ros.pdf

Panorama I

pp. 51-50 I

Volumen 9 |

Número 17 | Julio-diciembre | 2015 |
37. Zapata-Ros, M. (2013). MOOCs, una visión crítica y una alternativa complementaria: la individualización del aprendizaje y de la ayuda pedagógica. Campus Virtuales, 2(1), 20-38. 